\title{
Infectious diseases: surveillance, genetic modification and simulation
}

\author{
H. L. Koh ${ }^{1}$, S. Y. Teh ${ }^{2}$, D. L. DeAngelis ${ }^{3,4}$ \& J. Jiang ${ }^{4}$ \\ ${ }^{1}$ Disaster Research Nexus, School of Civil Engineering, \\ Universiti Sains Malaysia, Malaysia \\ ${ }^{2}$ School of Mathematical Sciences, Universiti Sains Malaysia, Malaysia \\ ${ }^{3}$ U.S. Geological Survey, Gainesville, Florida, USA \\ ${ }^{4}$ Department of Biology, University of Miami, Florida, USA
}

\begin{abstract}
Infectious diseases such as influenza and dengue have the potential of becoming a worldwide pandemic that may exert immense pressures on existing medical infrastructures. Careful surveillance of these diseases, supported by consistent model simulations, provides a means for tracking the disease evolution. The integrated surveillance and simulation program is essential in devising effective early warning systems and in implementing efficient emergency preparedness and control measures. This paper presents a summary of simulation analysis on influenza A (H1N1) 2009 in Malaysia. This simulation analysis provides insightful lessons regarding how disease surveillance and simulation should be performed in the future. This paper briefly discusses the controversy over the experimental field release of genetically modified (GM) Aedes aegypti mosquito in Malaysia. Model simulations indicate that the proposed release of GM mosquitoes is neither a viable nor a sustainable control strategy.

Keywords: dengue, A H1N1, GM mosquitoes, simulation.
\end{abstract}

\section{Introduction}

The World Health Organization (WHO) announced on 11 June 2009 that the influenza $\mathrm{A}(\mathrm{H} 1 \mathrm{~N} 1)$ has reached the stage known as pandemic alert phase 6 with moderate severity. On 10 August 2010, WHO declared that pandemic alert phase 6 for A $(\mathrm{H} 1 \mathrm{~N} 1)$ is officially over and the world has entered into the post pandemic period. However, the looming threat of another A (H1N1) pandemic 
remains. During the height of this A (H1N1) 2009 pandemic, there were concerns that more severe forms of A (H1N1) might emerge as second waves in the ensuing winter months. These second waves of disease might cause more people to be infected and might also induce higher fatality. This concern regarding the potential emergence of more potent second waves is premised upon the experience of the 1918-1919 Spanish Flu (A-H1N1), during which the second waves turned out to be more pervasive and destructive than the first [1]. Four decades later, two pandemics known as the Asian Flu (H2N2) of 1957-1958 and the Hong Kong Flu (H3N2) of 1968-69, resulted in deaths of about one million people. Having witnessed three pandemics in a span of a century, the last being forty years ago, the health care professionals have ample reasons to be alert to the potential of yet another pandemic of similar severity in the near future. At the height of the A (H1N1) epidemic in Malaysia in June 2009, the authors began to develop a flu simulation model known as FluSiM to track and understand the evolution of this epidemic. A simple model framework was chosen to reflect the degree of understanding of the A (H1N1) transmission dynamics, as well as to avoid the need for excessive medical data that were not available at that stage of disease development. The rational was to keep the model as simple as possible but not any simpler, consistent with the quality of data publicly available then (fig. 1). Further improvement is possible and indeed desirable in order to better utilize the predictive ability of the model. Figure 1 shows the number of new infected individuals on a weekly basis for the whole of Malaysia, beginning on week 24 and ending in week 50. The infection appeared to peak around week 33, about 60 days later. It is still not certain whether the cases reported on week 24 indeed started the subsequent infections. Hence, the time to peak might well be less than 60 days. A flu simulation model known as FluSiM is developed to fit the data for the A (H1N1) 2009 influenza for Malaysia. The bell shape curve of fig. 1 suggests that the popularly used model, based upon the concept of susceptible-infected-recovered (SIR), is appropriate and will be adopted in this paper.

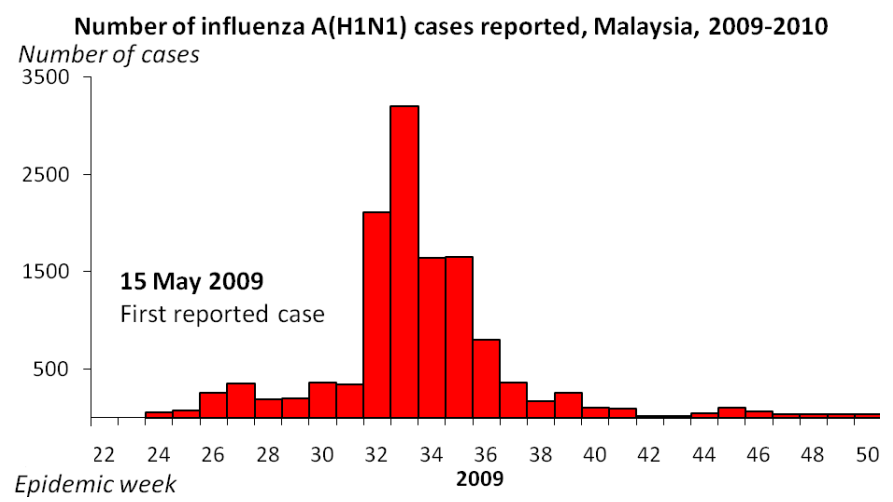

Figure 1: Weekly infected cases for Malaysia. 


\section{SIR model: FluSiM}

FluSiM is an SIR model developed to investigate the transmission dynamics of flu between susceptible individuals and infected individuals [2-4]. An infected individual is considered as infective. Central to this model framework of an infective disease epidemic for a given population is the assumption that new infective persons are generated by the mixing of uninfected susceptible persons with existing infective persons. Most epidemic models typically assume that the rate of increase of new infective individuals is proportional to the product of the number of susceptible individuals $(S)$ and the number of infective individuals $(I)$, shown as ( $\beta P s i)$ in eqs. (1) and (2). This is a well known mass action assumption, which has been successfully applied to a wide range of human and wildlife infectious diseases. This assumption implies that each susceptible and each infective are at all times equally accessible and exposed to each other. This underlying assumption of uniformity of exposure is normally not fully satisfied. An infective recovers after $T$ days $(T=1 / \alpha)$, after which he is no longer infective and is removed from the Infective compartment $(I)$ and moved to the Recovered

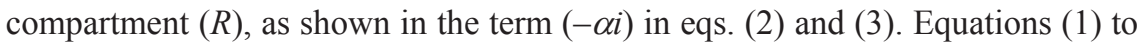
(3) constitute the SIR model with $s=S / P, i=I / P, r=R / P$ and $P=$ total population. Here, $S=$ number of Susceptibles, $I=$ number of Infectives, $R=$ number of Recovered and $P=S+I+R$.

$$
\frac{d s}{d t}=-\beta P s i \quad \text { (1) } \quad \frac{d i}{d t}=\beta P s i-\alpha i \quad \text { (2) } \quad \frac{d r}{d t}=\alpha i
$$

Early detection of epidemic of A (H1N1) requires real time data and real time simulation. Some variables are observable (e.g. infected persons I); some are hidden variables that are not observable (e.g. susceptible persons $S$ ). The mean duration of infectivity $T$ is an important parameter that hopefully could be reliably compiled during the course of the epidemic. On the other hand, the dynamics of transmission of A (H1N1) from the infective to the susceptible is not well understood. Further, direct measurement of the disease transmission rate is not possible. This transmission rate (known as contact rate $\beta P$ ) can only be estimated by model calibration. However, large variability and uncertainty are inherent in these unobservable variables such as the contact rate $\beta P$ and the total number of susceptible individuals $P$ in the model population.

An important concept in infectious disease transmission is the basic reproductive number, usually denoted by $R_{0}$. The basic reproductive number $R_{0}$ is defined as the average number of new infections that one infected individual generates in the susceptible population during the time that infected individual is infective [5]. For the SIR model, $R_{0}=\beta P / \alpha=\beta P T$. If $R_{0}$ is greater than 1 , an epidemic will occur; if $R_{0}$ is less than 1 , the outbreak will die down. A series of simulations was performed to test model sensitivity to input parameters $\alpha$ and $\beta P$, the results of which are plotted in fig. 2. The left figure shows the ratio $I / P$ (the proportion of susceptible that are eventually infected), while the right figure depicts the time to disease peak, as functions of $\alpha$ and $\beta P$. Based upon fig.1, the 

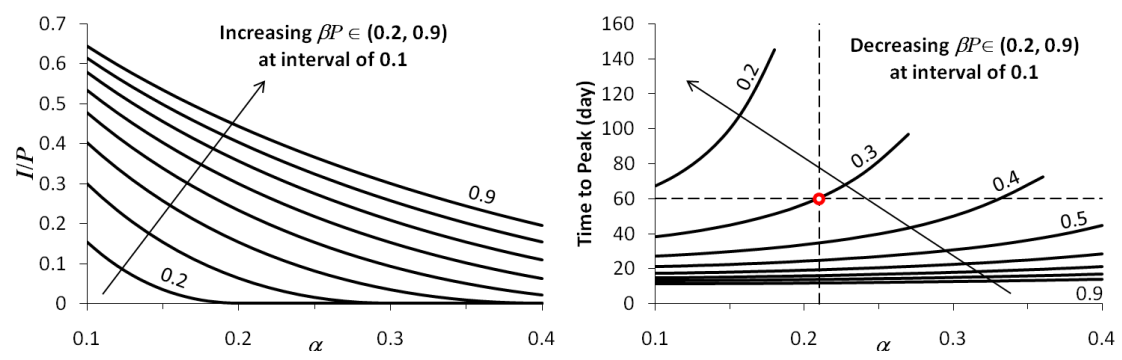

Figure 2: $\quad$ Dependency of FluSiM simulation results on $\alpha$ and $\beta P$.

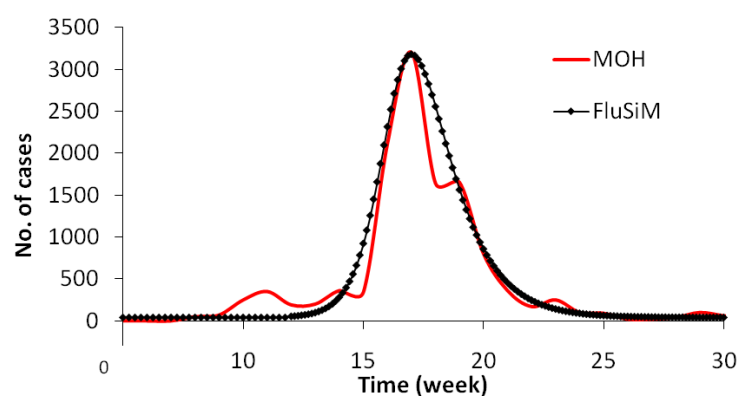

Figure 3: Comparison of $\mathrm{MOH}$ data and FluSiM, $\alpha=0.21, \beta P=0.3, R_{0}=$ 1.4 .

time to disease peak is about 60 days, which can be matched to various combinations of $\alpha$ and $\beta P$ in fig. 2 (right). Based upon literature search, the infective period $T$ for A (H1N1) varies generally between 3 to 7 days [4], or $\alpha$ between 0.1428 and $0.3333 \mathrm{~d}^{-1}$. Given the status of medical care in Malaysia, an infective period of about 5 days or $\alpha=0.2 \mathrm{~d}^{-1}$ is appropriate. The time to peak of 60 days can be fitted to the choice of $\alpha=0.21 \mathrm{~d}^{-1}$ and $\beta P=0.3$ with $R_{0}=\beta P T=$ 1.4. The infection curve for this choice is shown in fig. 3 , indicating reasonably good fit with $\mathrm{MOH}$ (Ministry of Health) data.

\section{Future surveillance and simulation program}

By fitting FluSiM model to A (H1N1) 2009 outbreak data for Malaysia, $R_{0}$ has been estimated to be around 1.4, which is comparable to the $R_{0}$ values of between 1.4 and 1.6 estimated by the SIR model for La Gloria in Mexico. This value is also comparable to the $R_{0}$ value of approximately 1.3 estimated for regular seasonal strain of influenza. For the 1918-1919 pandemic strain, $R_{0}$ has been estimated to be significantly higher, between 1.2 and 3.0 for community-based settling, and between 2.1 and 7.3 for confined settling [6]. The influenza A (H1N1) has been undergoing cross species evolution, and has been shown to have arisen from recombination of swine, avian and human strains. This continuous recombination may result in new strains, which may have high values 
of $R_{0}$, accompanied by high fatality rate, which could potentially inflict devastating medical consequences. Hence, we must establish an integrated surveillance and simulation program that is capable of detecting the eminent emergence of influenza that has the potential of inflicting severe consequences because of the high reproductive number $R_{0}$, accompanied by high fatality rate. The fatality rate is intimately related to the viral activities and properties, which is a domain of virologists. It is imperative that virologists should have the ability to isolate and identify flu viruses that have the potential of high fatality. High reproductive number $R_{0}$ may result from a combination of factors, including viral properties, mixing pattern of human population and demography. In particular, the duration $T$ of infectiveness of the disease plays an important role in determining the reproductive number since $R_{0}=\beta P T$. The contact rate $\beta P$ depends on both the transmission efficiency of the flu $\beta$ and the total number of susceptible $P$ in a given community. Estimating $\beta$ and $P$ requires real-time data and real-time model calibration, in addition to detailed surveillance of disease evolution. The accuracy of the SIR model is premised upon the uniformity of mixing between susceptible and infective individuals in the population. This uniformity may not be valid for large population living in big cities. Therefore, communities living in large cities such as Kuala Lumpur should be divided into sub-populations, to comply with the uniformity assumption, in order to improve the predictive capability of the SIR model.

\section{Controversy over genetically modified mosquito}

The Malaysia Institute of Medical Research (IMR), in collaboration with UKbased Biotech company Oxitec, has obtained approval from the Malaysian National Biosafety Board to conduct limited field trials of releasing genetically modified (GM) male mosquitoes (known as OX513A) to control the population of natural Aedes aegypti mosquitoes that carry the dengue virus. The GM male mosquitoes will mate with natural females to produce larvae that accumulate an extra enzyme to toxic levels, in the absence of the antibiotic tetracycline. This accumulated enzyme will eventually kill the larvae before they mature into adult mosquitoes, according to theory. These GM male mosquitoes will compete with natural male mosquitoes to mate with natural females. In theory, repeated releases of GM males would reduce natural mosquito population in dengue prone areas. However, the natural mosquito will grow back to their natural population level after the discontinuation of GM mosquito release. The mosquitoes will be released in the inland districts of Bentong in Pahang and Alor Gajah in Malacca, according to the National Biosafety Board. The release in each location will proceed in two phases: the first at a site at least half a kilometre from the nearest human settlement, and the second in an inhabited site. For each experimental release, an estimated 4000 to 6000 male GM mosquitoes are expected to be released, along with similar number of natural male A. aegypti mosquitoes. These experiments will be repeated over several months. Mosquitoes in the release sites will be captured using mosquito traps, and will be monitored for at 
least one month. At the end of the field trial, fogging in a $400 \mathrm{~m}$ radius is required. A second fogging should be conducted one week after the first fogging.

This planned controversial field experiments have aroused considerable anxiety among NGOs and concerned scientists, primarily due to the lack of transparency, the absence of meaningful and effective public participation, and the seeming haste in the approval process. Serious ethical, legal, public health and human rights issues involved in these experiments have not been sufficiently addressed by the Malaysian approval authorities. Lab tests had shown that three to four percent of the offspring of male GM mosquitoes mating with normal females do actually survive into adulthood, contrary to what has been falsely claimed that all GM larvae will die as intended. This residual mosquito will eventually grow in population size quickly due to their fast development rate. Further, female GM mosquitoes might accidentally be released. The males are separated from the female GM mosquitoes based on the size of the pupae. This separation process is not completely safe-proof. Because of the controversy, the IMR has yet to fix a new release date, having failed to initiate the field experiment as planned earlier. The IMR and Oxitec have cited the reported success of similar experiments conducted in the Cayman Island to justify their field experiment. The reported success in the Cayman Island should not be used to project success of similar experiment in Malaysia, as the geography of the two sites are vastly different. While Cayman Island (land area $200 \mathrm{~km}^{2}$ ) is small and isolated from populations by the surrounding sea, Malaysia (land area 330,000 $\mathrm{km}^{2}$ ) is a large country surrounded by many countries with huge populations. This geographical connectivity allows mosquito free range of movement over time, thereby permitting natural mosquito to invade areas previously rid of mosquito due to the GM experiment. This ability of natural mosquitoes to reinvade regions previously void of mosquitoes will be demonstrated in our simulations.

\section{Simulation model for GM mosquito}

This paper investigates by model simulations the efficacy of controlling natural Aedes aegypti mosquito population by the release of GM mosquitoes into the natural habitat. Esteva and Yang [7] proposed a mathematical model (4)-(9) to assess the effectiveness of controlling mosquitoes by the sterile insect technique. Their modelling approach is adapted in this paper to investigate the efficacy of releasing GM mosquito into a natural habitat to eradicate natural mosquito populations as a way to control mosquito-borne diseases. In this model, the life cycle of a mosquito is divided into two stages: the immature aquatic form (eggs, larvae and pupae) and the adult winged form $[8,9]$. We denote by $A$ the immature aquatic form of the mosquito. The adult wing mosquitoes are divided into five compartments. The first compartment consists of unmated single females, which is denoted by $F_{s}$. Some of these single unmated females remain unmated and therefore remain in $F_{s}$, while the remaining singles are mated. The mated females are further divided into two compartments. Those that are 
Table 1: $\quad$ Parameter values used for simulation study $[7,10]$.

\begin{tabular}{|c|c|c|c|c|c|}
\hline$\mu_{A}$ & $\mu_{S}$ & $\mu_{f}$ & $\mu_{u}$ & $\mu_{M}$ & $\mu_{T}$ \\
\hline 0.05 & 0.05 & 0.05 & 0.05 & 0.1 & 0.1 \\
\hline$\phi$ & $\gamma$ & $r$ & $\beta$ & $C$ & $p q$ \\
\hline 5.0 & 0.075 & 0.5 & 1.0 & 600 & 0.7 \\
\hline
\end{tabular}

fertilized are moved into compartment denoted by $F_{f}$, while the unfertilized females are moved to the compartment $F_{u}$. Natural male mosquitoes form members of the compartment denoted by $M$. Finally, the GM male mosquito is grouped into compartment denoted by $M_{T}$. This set of compartment notations will be used in this paper. For example, the per capita mortality rates of the immature aquatic form, unmated single females, mated fertilized females, mated unfertilized females, natural male and GM male mosquitoes are denoted by $\mu_{A}$, $\mu_{s}, \mu_{f}, \mu_{u}, \mu_{M}$ and $\mu_{T}$, respectively. This GM simulation model will be run with input parameters shown in table 1. Details of the model can be referred to [7].

$$
\begin{aligned}
& \frac{d A}{d t}=\phi\left(1-\frac{A}{C}\right) F_{f}-\left(\gamma+\mu_{A}\right) A \\
& \frac{d F_{s}}{d t}=r \gamma A-\frac{\beta M F_{s}}{M+M_{T}}-\frac{\beta_{T} M_{T} F_{s}}{M+M_{T}}-\mu_{s} F_{s} \\
& \frac{d F_{f}}{d t}=\frac{\beta M F_{s}}{M+M_{T}}-\mu_{f} F_{f}
\end{aligned}
$$

$$
\begin{aligned}
& \frac{d F_{u}}{d t}=\frac{\beta_{T} M_{T} F_{s}}{M+M_{T}}-\mu_{u} F_{u} \\
& \frac{d M}{d t}=(1-r) \gamma A-\mu_{M} M \\
& \frac{d M_{T}}{d t}=\alpha-\mu_{T} M_{T}
\end{aligned}
$$

$A=$ population of the aquatic forms of mosquito;

$F_{s}=$ population of winged females before mating (singles);

$F_{f}=$ population of mated fertilized winged females;

$F_{u}=$ population of mated unfertilized winged females;

$M=$ population of winged natural males mosquito;

$M_{T}=$ population of winged transgenic GM males mosquito;

$\gamma=$ development rate of aquatic form, day ${ }^{-1}$;

$\beta=$ mating rate of natural mosquito, day $^{-1}$;

$\alpha=$ number of winged transgenic males released per day, mosquito day ${ }^{-1}$;

$\phi=$ oviposition rate of fertilized winged females, day $^{-1}$;

$C=$ carrying capacity of aquatic forms of mosquito;

$r=$ ratio of aquatic forms that becomes winged females, mosquito/mosquito;

$\beta_{T}=p q \beta=$ mating rate of transgenic mosquito, day $^{-1}$;

$p=$ ratio of transgenic males to natural females, mosquito/mosquito;

$q=$ ratio of effective mating rate of transgenic males, day ${ }^{-1} /$ day $^{-1}$;

$\mu_{A}=$ mortality rate of aquatic forms, day $^{-1}$;

$\mu_{s}=$ mortality rate of winged females before mating, day ${ }^{-1}$;

$\mu_{f}=$ mortality rate of mated fertilized winged females, day ${ }^{-1}$;

$\mu_{u}=$ mortality rate of mated unfertilized winged females, day ${ }^{-1}$; 
$\mu_{M}=$ mortality rate of winged natural males, day ${ }^{-1}$;

$\mu_{T}=$ mortality rate of winged transgenic males, day ${ }^{-1}$.

\section{Simulation results for GM mosquito}

The parameter values used in the simulation study are listed in table 1 . We further assume that $\beta_{T} / \beta=p \times q=0.7$, which implies that GM males lose $30 \%$ of the natural mating capacity due to imperfect physiological modifications following GM process and less than optimal choice of release site resulting in reduced mating success [7]. Additional details regarding $p q$ will be discussed in a later section. To initiate mosquito population, we choose an initial population consisting of only aquatic form with density equivalent to $90 \%$ of carrying capacity of aquatic form (denoted by $A / C=0.9$ ). This choice of initial population is appropriate as the GM release is conducted in areas infested with Aedes mosquito. It is not necessary to have initial population of adult mosquitoes, as the natural aquatic forms will mature into adult forms. Inclusion of initial adult forms into the model framework would not change any conclusions reached in this paper. A large initial population of GM male mosquitoes 20 times the carrying capacity of aquatic forms $\left(M_{T} / C=20\right)$ is assumed to be released into the experimental site at the start of the simulation. Other initial release conditions will also be simulated for sensitivity analysis. In section 6.1 , we consider the case with completely enclosed release site, where mosquitoes will not be recruited from external sources. Then in section 6.2, we consider the case with porous release site, which is open to its neighbour, in which case mosquitoes will be recruited from external sources by natural diffusion and advection.

\subsection{Completely enclosed release site}

We first consider the case in which the release site is assumed to be completely isolated from its neighbours, with no possibility of mosquito recruits coming from external regions. Various scenarios are simulated. The first scenario considers a continuous release of GM male mosquitoes (equivalent to $\alpha=75$ mosquitoes/day) into this enclosed release site, in addition to the initial large one-time release of GM males $\left(M_{T} / C=20\right)$ mentioned earlier. The second scenario considers no continuous release of GM male mosquitoes ( $\alpha=0$ mosquitoes/day) after the initial one-time release. Simulation results for these two scenarios are compared in fig. 4. The initial one-time release of GM male mosquitoes will quickly decrease the number of aquatic forms, as the offspring produced by the female mosquitoes that mated with GM male mosquitoes are killed during the aquatic phase before they can develop into mature form. Hence, the adult form will be decimated. However, without continuous release of GM male mosquitoes after the initial one-time release, the GM male mosquitoes released initially will eventually die. The residual mosquito population in the enclosed site will then recover to their normal population levels in the absence of GM males. It is assumed that both GM and natural males have the same mortality rates. On the other hand, if there is a continuous unending release of 


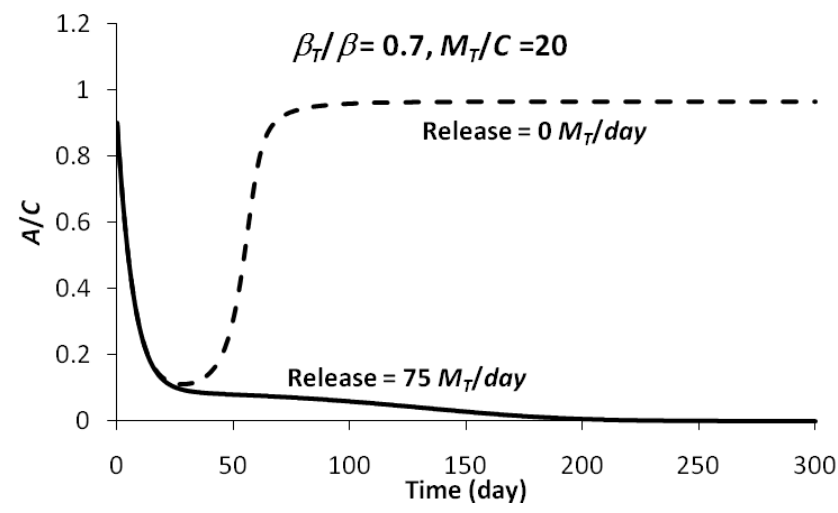

Figure 4: Proportion $A / C$ subject to one initial release and continuous release of GM mosquitoes.

GM male mosquitoes, the natural mosquitoes will be wiped out eventually, at the indicated rates of initial and continuous release. We next consider reduced rates of initial release of GM males.

The third scenario considers several cases with reduced one-time initial release of GM mosquito equivalent to 1, 4, 10, 15 and 20 times the carrying capacity of aquatic forms (C), and with the same continuous release of 75 mosquitoes per day. This sensitivity analysis indicates that the natural mosquito population will initially decrease to lower levels due to the large initial one-time release of GM males. The higher the initial one-time release rate, the sharper will be the initial mosquito population depression. However, with the exception of release rate equivalent to 20 times $\mathrm{C}$, the natural mosquito population will eventually grow back to their natural population level. Only the time taken to recover to the natural population level varies. The higher the initial one-time release rates, the longer will be the time taken to recover to the natural state (fig. 5). In short, the proposed GM mosquito release program is not a viable strategy to eradicate natural A. aegypti mosquitoes, as the natural mosquito population will persist in this enclosed release site.

Finally, we investigate the role of GM male mating success in reducing natural mosquito population. For this purpose, two critical parameters are relevant. First, the parameter $p(0 \leq p \leq 1)$ refers to the relative effectiveness of GM male mosquitoes in searching for female natural mosquitoes for mating. Values of $p$ are sensitive to the environment into which the GM male mosquito is being released $[11,12]$. The parameter $p$ plays an important role in reproduction success of GM males in a habitat with varying contact rates with natural female mosquito. Next, the parameter $q(0 \leq q \leq 1)$ may be thought of as a measure of reduced efficiency in mating success due to physiological imperfection induced by the genetic modification process. Sensitivity analysis is performed to assess the combined impact of $\beta_{T} / \beta=p q$ on the persistence of the natural mosquito population subject to the release of GM males, the result of which is presented in fig. 6 . For this purpose, we first assume a $16 \%$ reduction in mating success of 

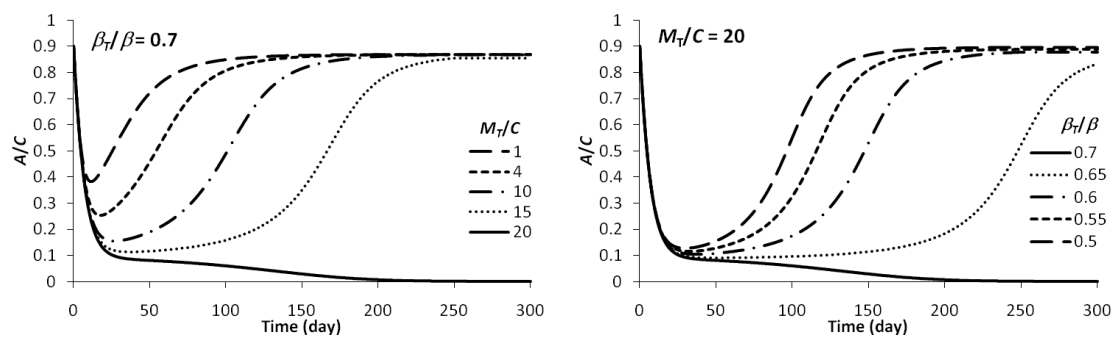

Figure 5: Proportion $A / C$ for Figure 6: several $M_{T} / C$.

Proportion $A / C$ for several $\beta_{T} / \beta$.

GM males due to imperfection of genetic modification. Further, we also assume a $16 \%$ reduction in the success of choosing the optimal release site. This choice results in $p \times q=0.84 \times 0.84=0.70$. A large initial one-time GM release $\left(M_{T} / C=\right.$ 20) followed by a continuous daily release of GM males (equivalent to $\alpha=75$ mosquitoes/day) discussed earlier and subject to $p q=0.70$ will wipe out the natural population eventually. However, reducing $p q$ to 0.65 or lower will allow the natural population to eventually recover and persist in its natural population level even in the presence of GM mosquitoes. To perpetually maintain a value of $p q \geq 0.7$ remains a goal that is not viable to achieve under normal field conditions and subject to imperfect genetic modification. Hence, the strategy of releasing GM male mosquitoes to control natural A. aegypti population is not viable, based upon model simulations performed in this paper.

\subsection{Porous release site}

Physically connected to external areas, a natural release site is neither completely enclosed nor completely isolated from its neighbours. Hence, mosquitoes from external sources will be able to invade the release site over time. We therefore simulate the propagation of mosquito travelling wave in an extended natural habitat covering tens of kilometres. The wave is initiated by a small initial population equivalent to $1 \%$ of the carry capacity at the location $X=0 \mathrm{~km}$. We assume that the mosquito disperse in space in search for meals and breeding grounds, assisted by natural atmospheric diffusion, which is modelled by a dispersion coefficient $D$ of $0.013 \mathrm{~km}^{2} / \mathrm{d}[8,13]$. In some situation, wind might also help to transport mosquito over space, which is modelled by an advection coefficient $v$ of $0.1 \mathrm{~km} / \mathrm{d}$. Figure 7 (left) shows the symmetrical spread of mosquito in both directions from its small initial population at $\mathrm{X}=0 \mathrm{~km}$, given the values of $D=0.013 \mathrm{~km}^{2} / \mathrm{d}$ and $v=0 \mathrm{~km} / \mathrm{d}$. The mosquito population advances symmetrically in both directions with travelling wave velocity $\lambda$ of 0.3 $\mathrm{km} / \mathrm{d}$. Figure 7 (right) shows the asymmetrical spread of mosquito from its small initial population at $\mathrm{X}=0 \mathrm{~km}$, given the values of $D=0.013 \mathrm{~km}^{2} / \mathrm{d}$ and $v=0.1$ $\mathrm{km} / \mathrm{d}$. The mosquitoes propagate in the downwind direction with travelling wave of $\lambda=0.4 \mathrm{~km} / \mathrm{d}$ and in the upwind direction with $\lambda=0.2 \mathrm{~km} / \mathrm{d}$. Based upon these simulation results, it may be concluded that mosquito might readily invade any 

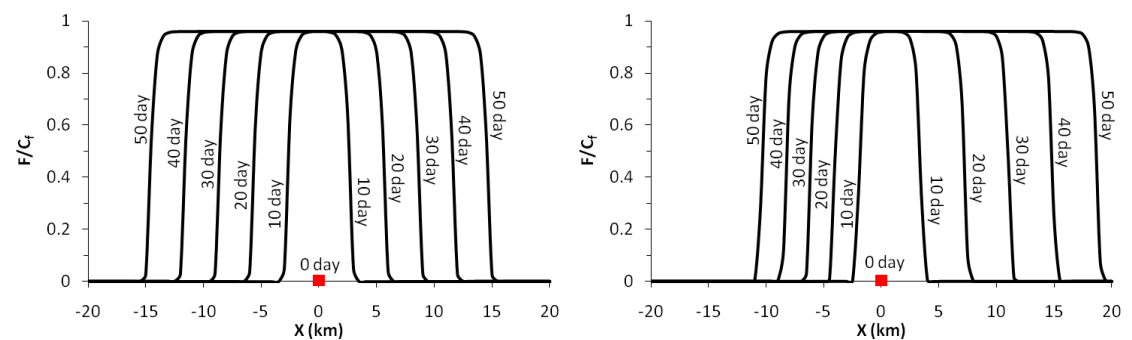

Figure 7: Mosquito traveling waves in a natural environment with no advection (left) and with advection towards the east (right).

habitat with travelling wave velocity of the order of $\lambda=0.1 \mathrm{~km} / \mathrm{d}$. A geographical separation of the order of one or two $\mathrm{km}$ provides inadequate resistance to mosquito invasion. Hence, the strategy of GM male release to control natural mosquito population is not sustainable in the long run.

\section{Conclusion}

The simple SIR model is suitable for the simulation of influenza A (H1N1) 2009 in Malaysia. To prepare for a scenario with very severe form of influenza in the future, an integrated surveillance and simulation program is essential. A large community living in big cities must be divided into sub-populations in this SIR model in order to improve predictive capability of the model. The planned release of the genetically modified male mosquitoes into natural habitat to control mosquito population has aroused considerable attention. Simulation results indicate that the release of GM male mosquitoes to control natural Aedes aegypti population is neither viable nor sustainable.

\section{Acknowledgements}

Financial support provided by Grants 1001/PMATHS/817024, 1001/PMATHS/817025, 1001/PMATHS/811093 and 203/PMATHS/671187 is gratefully acknowledged.

\section{References}

[1] Massad, E., Burattini, M.N., Coutinho, F.A.B. \& Lopez, L.F., The 1918 influenza A epidemic in the city of São Paulo, Brazil. Medical Hypotheses, 68(2), 2007, pp. 442-445.

[2] Towers, S. \& Feng, Z., Pandemic H1N1 influenza: Predicting the Course of a Pandemic and Assessing the Efficacy of the Planned Vaccination Programme in the United States, Rapid communications. Eurosurveillance, 14(1), pp. 1-3, 2009. 
[3] Cruz-Pacheco, G., Duran, L., Esteva, L., Minzoni, A.A., López-Cervantes, M., Panayotaros, P., Ahued Ortega, A. \& Villaseñor Ruíz, I., Modelling of the influenza A(H1N1)v outbreak in Mexico City, April-May 2009, with control sanitary measures, Rapid communications. Eurosurveillance, 14(26), pp. 1-3, 2009.

[4] Koh, H.L. \& Teh, S.Y., Simulation of A H1N1 2009 in Malaysia. Proc. of the 2010 3rd International Conference on Environmental and Computer Science (ICECS 2010), Volume 1, 17-19 October 2010, Kunming, China, International Association of Computer Science \& Information Technology (IACSIT), IEEE Computer Society, pp. 33-37, 2010.

[5] Boni, M., Bui, H.M., Pham, Q.T., Farrar, J., Tran, T.H., Nguyen, T.H., Nguyen,V.K.\& Horby, P., Modelling the progression of pandemic influenza A (H1N1) in Vietnam and the opportunities for reassortment with other influenza viruses. BMC Medicine, 7(1), pp. 43, 2009.

[6] Vynnycky, E., Trindall, A. \& Mangtani, P., Estimates of the reproduction numbers of Spanish influenza using morbidity data. International Journal of Epidemiology, 36(4), pp. 881-889, 2007.

[7] Esteva, L. \& Yang, H.M., Mathematical model to assess the control of Aedes aegypti mosquitoes by the sterile insect technique. Mathematical Biosciences, 198(2), pp. 132-147, 2005.

[8] Koh, H.L., Teh, S.Y., Izani, A.M.I. \& DeAngelis, D.L., Modeling Biological Invasion: The Case of Dengue and Mangrove. Proc. of the International Conference on Mathematical Biology (ICMB07), 4-6 September 2007, Kuala Lumpur, Malaysia, ed. K.A. Mohd. Atan. American Institute of Physics Conference Proceedings 971(1), New York, pp. 11-18, 2008.

[9] Koh, H.L., Teh, S.Y., Majid, T.A. \& Abdul Aziz, H., Grid Computing for Disaster Mitigation. In: Data Driven e-Science: Use Cases and Successful Applications of Distributed Computing Infrastructures (ISGC 2010), eds. S.C. Lin and E. Yen, Springer, New York, in press.

[10] Gubler, D.J., Dengue, The Arboviruses: Epidemiology and Ecology, vol. II, ed. T.P. Monath, CRC, Boca Raton, FL, p. 213, 1986.

[1] Moreira, L.A., Ghosh, A.K., Abraham, E.G. \& Jacobs-Lorena, M., Genetic transformation of mosquitoes: a quest for malaria control, Invited review. International Journal for Parasitology, 32(13), pp. 1599-1605, 2002.

[11] Rafikov, M., Bevilacqua, L. \& Wyse, A.P.P., Optimal control strategy of malaria vector using genetically modified mosquitoes. Journal of Theoretical Biology, 258(3), pp. 418-425, 2009.

[12] Takahashi, L.T., Maidana, N.A., Ferreira, Jr., W.C., Pulino, P. \& Yang, H.M., Mathematical models for the Aedes aegypti dispersal dynamics: travelling waves by wing and wind. Bulletin of Mathematical Biology, 67(3), pp. 509-528, 2005. 